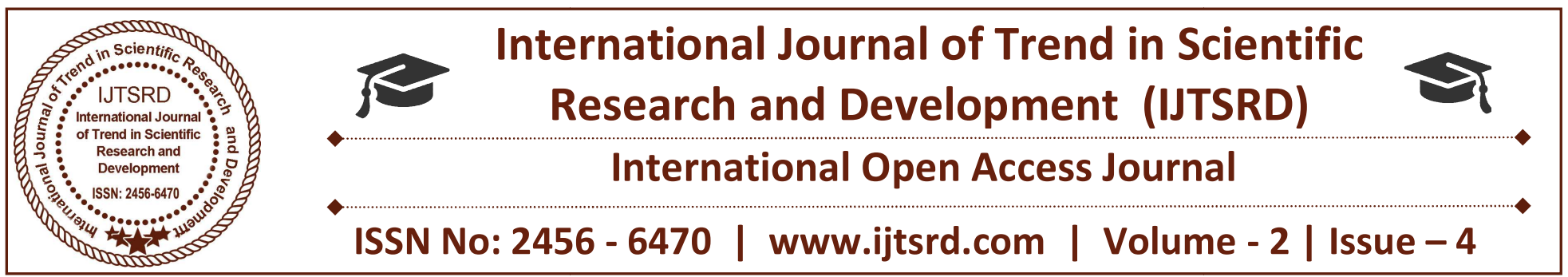

\title{
Data Outsourcing with Secure Data Auditing in Cloud Computing
}

\author{
J. Gopinadh \\ MCA Final Year, Lakireddy Bali Reddy College Of Engineering, Mylavaram, Andhra Pradesh, India
}

\section{ABSTRACT}

An ever increasing number of customers might want to store their information to public cloud servers (PCSs) alongside the quick improvement of distributed computing. New security issues must be understood keeping in mind the end goal to enable more customers to process their information in broad daylight cloud. Right when the client is bound to get to PCS, he will name its middle person to process his data and exchange them. On the other hand, remote data trustworthiness checking is furthermore a basic security issue with no attempt at being subtle disseminated stockpiling. It impacts the clients to check whether their outsourced data is kept set up without downloading the whole data. From the security issues, we propose a novel intermediary situated information transferring and remote information uprightness checking model in personality based open key cryptography: character based intermediary arranged information transferring and remote information respectability checking in broad daylight cloud. We give the formal definition, framework model, and security demonstrates. At that point, a convention is outlined utilizing the bilinear pairings. The proposed convention is provably secure in view of the hardness of computational DiffieHellman issue. Our convention is likewise efficient and flexible. In light of the first customer's approval, the proposed convention can understand private remote information uprightness checking.

Keywords: Security, Encryption algorithm, PCS, PKG

\section{INTRODUCTION}

Identity based public key system (ID-PKS) is an option for open key cryptography. ID-PKS setting kills the requests of public key infrastructure (PKI) and certificate organization in regular open key settings. An ID-PKS setting includes confided in outcast (i.e. private key generator, $\mathrm{PKG}$ ) and a customers. The PKG is skilled to convey every client private key by utilizing the related ID data (e.g.name, email address, or standardized speculation stores number). As necessities the recipient utilizes private key with ID to unwind such substance. An open key setting needs to give a client denial fragment, there look issue on the best way to deal with deny getting rowdy or traded off clients in PKS setting is normally expanded. In customary open key settings, certificate list is a known approach. In this approach, if a gettogether gets an open key and its related certificate, she/he first favors them and after that pivots toward the sky the CRL to guarantee comprehensive group key have not been denied. In such a case, the methodology needs the online support, so it will cause related bottleneck. To enhance the procedure execution, two or three capable repudiation portions for standard open setting has been for PKI. Unmistakably, agents also revolve around the repudiation issue of ID-PKS Settings.

\section{Related Work}

This region covers review of trademark based encryption; ID-PKS is a probability for open key cryptography. PKS settings dispose of the requesting of PKI and certificate relationship in standard key settings. An ID-PKS setting includes trusted in outsider and user's. The PKG is mindful so as to make each client's private key by utilizing the related ID information. The certificate and PKI are over the top in the same cryptographic parts under PKS settings. 
ID based encryption permitting as to encode messages especially by utilizing ID without checking the support of open key certificates. As the recipient utilizing the private key related with their comparing ID to decipher such figure substance. Since an open key setting needs to give a client renouncement fragments and the examination issue on the most able method to traded off clients in an ID-PKS settings. In this cases, the framework requires the online web help with comparing PKI, so it will acknowledge related bottleneck. To update the execution, a few powerful denial structures for typical open key settings have been we examined for PKI. Frankly, analysts moreover base on the repudiation issue of ID-PKS settings cryptography is signify decision for laying out cryptographic frameworks. Cryptography is art of disguising data from unintended person. There are two sorts of cryptographic structures one is 'Symmetric Cryptography' and other one is called as 'Open Key Cryptography'[08]. Symmetric cryptography uses single key for both encryption and unscrambling however open key cryptosystems uses two keys that are deductively associated (open key for encryption and private key for deciphering).

\section{Proposed Work}

Differentiated and the previous works, our arrangement does not have to re-issues total private keys, just need to revive a small weight section remembering the true objective to handle both the unadaptability and the inefficiency we will propose another revocable IBE plot with (CRA). With the guide of CRA, customer needs not to contact key invigorate, in a manner of speaking, $\mathrm{PKG}$ is allowed to be disengaged in the wake of sending the refusal summary to CRA. No sheltered channel or customer affirmation is required in the midst of key-revive among customer and CRA. Finally, to give expansive exploratory results to demonstrate the viability of our proposed advancement.

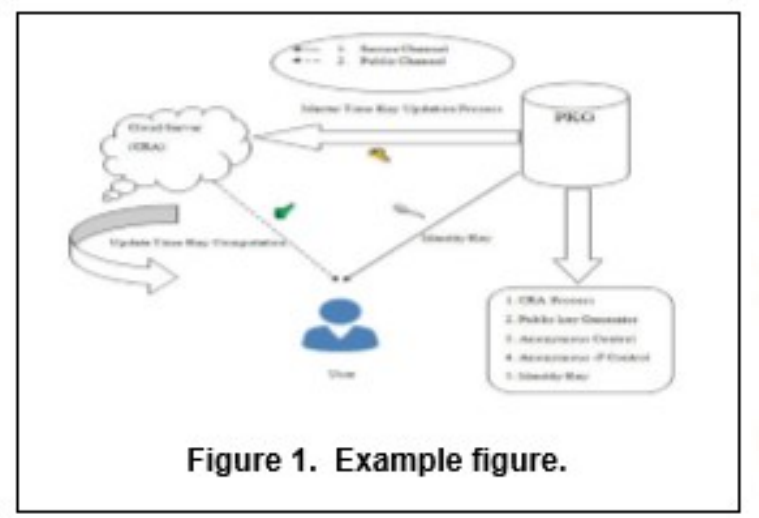

\section{Conclusion}

The inspected assertions of utmost in distributed computing information in a multi client. Shown the likelihood of same related based information out sourcing and proposes an IBDO conspire. It engages the lower to consign outsourcing capacity to delegates the information. Simply the affirmed mediator process and outsources for the advantage of the le-proprietor. Both the reason and respectability can be moved by an open analyst. The procedure of character based portion and the thorough investigating highlight make our course of action productive existing POR/PDP plans. The Security examinations and test comes to show the proposed plot is secure and has meet execution as SW design.

\section{References}

1) J. Yu, K. Ren, C. Wang, and V. Varadharajan, "Enabling cloud storage auditing with keyexposure resistance," Information Forensics and Security, IEEE Transactions on, vol. 10, no. 6, pp. 1167-1179, June 2015.

2) Y. Wang, Q. Wu, B. Qin, X. Chen, X. Huang, and J. Lou, "Ownership-hidden group-oriented proofs of storage from pre- homomorphic signatures," Peer-to-Peer Networking and Application- s, pp. 1-17, 2016.

3) T. Jiang, X. Chen, and J. Ma, "Public integrity auditing for shared dynamic cloud data with group user revocation," IEEE Transactions on Computers, vol. 65, no. 8, pp. 2363-2373, Aug 2016.

4) X. Fan, G. Yang, Y. Mu, and Y. Yu, "On indistinguishability in remote data integrity checking," The Computer Journal, vol. 58, no. 4, pp. 823-830, 2015. 
5) Adi Shamir, 'Identity-based cryptosystems and signature schemes," In Crypto 84, Springer, 1985.

6) W. Diffie and M. Hellman, "New directions in cryptography," IEEE Transactions on Information Theory, 1976.

7) S. Galbraith,I. Blake, G. Seroussi and N. Smart, "Advances in Elliptic Curve Cryptography," Cambridge University Press , 2005.

8) Fuchun Guo, Yi Mu, "Optimized Identity-Based Encryption from Bilinear Pairing for Lightweight Devices",in Proc. IEEE TRANSACTIONS ON DEPEND-ABLE AND SECURE COMPUTING, VOL. 14, NO. 2, MARCH/APRIL 2017.

9) Atkin and F. Morain, "Elliptic curves and primality proving," Mathematics of Computation, vol. 19, 1993.

10) Imperial Journal of Interdisciplinary Research (IJIR) Peer Reviewed - International Journal Vol4, Issue-1, 2018 (IJIR)

11) P. Barreto, S. Galbraith, C. and M. Scott, "E cient pairing computation on super singular abelian varieties," Designs, Codes and Cryptography, 2007.

12) P. Barreto, H. Kim, B. Lynn and M. Scott, "Efficient algorithms for pairing- based cryptosystems," Advances in Cryptology CRYPTO 2002, Lecture Notes in Computer Science, 2002.

13) Dan Boneh, "The decisional diffie-hellman problem," In Third Algorithmic Number Theory Symposium, pages 4863. Springer-Verlag, 1998.

14) Whitfield Diffie and Martin E. Hellman, "new directions in cryptography," IEEE Transactions on Information Theory, IT-22(6):644654, 1976.

15) K. D. Bowers, A. Juels, and A. Oprea, "HAIL: A High-availability and Integrity Layer for Cloud Storage," in Proceedings of the $16^{\text {th }}$ ACM Conference on Computer and Communications Security. New York, NY, USA: ACM, 2009, pp. 187-198.

16) K. G. Paterson and J. C. N. Schuldt, "Efficient Identity-Based Signatures Secure in the Standard Model," in Information Security and Privacy, ser. LNCS, L. Batten and R. Safavi-Naini, Eds., vol. 4058. Springer, Heidelberg, 2006, pp. 207-222.

17) D. Boneh, B. Lynn, and H. Shacham, "Short Signatures from the Weil Pairing," in Advances in
Cryptology-ASIACRYPT 2001, vol. 2248. Springer, Heidelberg, 2001, pp. 514-532.

18) B. Waters, "Efficient identity-based encryption without random oracles," in Advances in Cryptology-EUROCRYPT 2005, ser. LNCS, R. Cramer, Ed. Springer Berlin Heidelberg, 2005, vol. 3494, pp. 114-127.

\author{
(a)
}

